\title{
Accurate modeling of the hose instability in plasma wakefield accelerators
}

Cite as: Phys. Plasmas 25, 056703 (2018); https://doi.org/10.1063/1.5017960

Submitted: 01 December 2017 . Accepted: 20 February 2018 . Published Online: 20 March 2018

T. J. Mehrling (D, C. Benedetti, C. B. Schroeder, A. Martinez de la Ossa (D), J. Osterhoff, E. Esarey, and W.

P. Leemans (D)

\section{ARTICLES YOU MAY BE INTERESTED IN}

Comparative study of active plasma lenses in high-quality electron accelerator transport lines

Physics of Plasmas 25, 056702 (2018); https://doi.org/10.1063/1.5018001

Frontiers of beam diagnostics in plasma accelerators: Measuring the ultra-fast and ultra-cold Physics of Plasmas 25, 056704 (2018); https://doi.org/10.1063/1.5017847

Laboratory space physics: Investigating the physics of space plasmas in the laboratory Physics of Plasmas 25, 055501 (2018); https://doi.org/10.1063/1.5025421

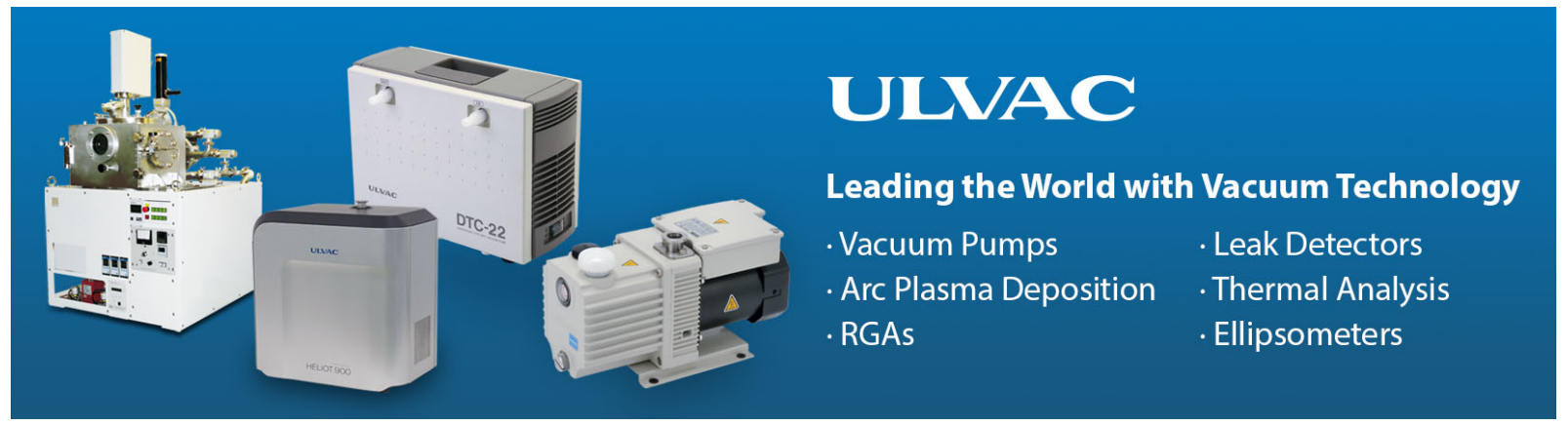




\title{
Accurate modeling of the hose instability in plasma wakefield accelerators
}

\author{
T. J. Mehrling, ${ }^{1,2, a), b)}$ C. Benedetti, ${ }^{2}$ C. B. Schroeder, ${ }^{2}$ A. Martinez de la Ossa, ${ }^{3}$ \\ J. Osterhoff, ${ }^{\prime}$ E. Esarey, ${ }^{2}$ and W. P. Leemans ${ }^{2}$ \\ ${ }^{1}$ Deutsches Elektronen-Synchrotron DESY, 22607 Hamburg, Germany \\ ${ }^{2}$ Lawrence Berkeley National Laboratory, Berkeley, California 94720, USA \\ ${ }^{3}$ Institut für Experimentalphysik, Universität Hamburg, 22761 Hamburg, Germany
}

(Received 1 December 2017; accepted 20 February 2018; published online 20 March 2018)

Hosing is a major challenge for the applicability of plasma wakefield accelerators and its modeling is therefore of fundamental importance to facilitate future stable and compact plasma-based particle accelerators. In this contribution, we present a new model for the evolution of the plasma centroid, which enables the accurate investigation of the hose instability in the nonlinear blowout regime. It paves the road for more precise and comprehensive studies of hosing, e.g., with drive and witness beams, which were not possible with previous models. Published by AIP Publishing.

https://doi.org/10.1063/1.5017960

\section{INTRODUCTION}

Plasma wakefield accelerators (PWFAs) ${ }^{1,2}$ allow for an energy gain of several GeVs within distances of only tens of centimeters for electron beams ${ }^{3,4}$ and therefore provide a transformative potential for the miniaturization of future particle acceleration facilities. For applications driven by such compact accelerators, the beam stability is of utmost importance. In this context, the extreme focusing fields, which can be on the same order of magnitude as the accelerating fields, entail the challenge of rapid growth rates for transverse beam-plasma instabilities. In a seminal work in the early 1990 s, the hose instability was predicted to pose a fundamental impediment for stable acceleration of beams in PWFAs. ${ }^{5}$ Hosing is also a challenge for long-beam PWFAs. ${ }^{6}$

Of specific interest for short-beam PWFAs is the nonlinear blowout regime, ${ }^{7}$ for which essentially all electrons are expelled from the vicinity of the drive beam and a co-propagating ion cavity is formed. The focusing fields in this regime are linear and the accelerating field enhanced. Electrons in the sheath, which surrounds the ion-channel, can have relativistic velocities and the blowout radius can be greater than the charge neutralization radius, assumed for the treatment in Ref. 5. As a result of these effects, the coupling between beam and plasma can be less enhanced, such that the growth rate for hosing is reduced. ${ }^{8}$ While hosing was still predicted to be growing exponentially in time and along the beam by previous models, ${ }^{5,8}$ a recent work showed that the intrinsic energy evolution and/or energy spread of the beam lead to a saturation or damping of hosing in PWFAs in the blowout regime. ${ }^{9}$ This is similar to the mitigation of hosing in self-modulated PWFAs owing to a varying betatron wavenumber along the beam $^{10}$ and similar to Balakin-NovokhatskySmirnov damping in conventional accelerators. ${ }^{11}$

The hose instability occurs when transverse phase space asymmetries of the beam coherently couple with phase space

\footnotetext{
Note: Paper YI3 4, Bull. Am. Phys. Soc. 62, 405 (2017)

${ }^{a)}$ Invited speaker.

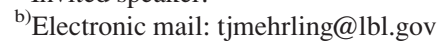

asymmetries of the plasma. For example, a displacement of the transverse beam centroid $X_{b}$ from the propagation axis induces a deviation of the plasma wake centroid $X_{p}$ along the beam, which then feeds back to the temporal evolution of the trailing part of the beam. A mathematical description of the hose instability in PWFAs is given by the coupled differential equations 8,9

$$
\begin{gathered}
\frac{\partial^{2} X_{p}}{\partial \xi^{2}}+\frac{c_{\psi}(\xi) c_{r}(\xi)}{2}\left(X_{p}-X_{b}\right)=0, \\
\frac{\partial^{2} X_{b}}{\partial t^{2}}+\lambda(\xi, t) \frac{\partial X_{b}}{\partial t}+\Omega^{2}(\xi, t)\left(X_{b}-X_{p}\right)=0,
\end{gathered}
$$

where $\xi=t-z$ is the co-moving variable, $z$ the propagation distance, and $t$ the time. Length-scales within this work are normalized by the plasma wavenumber $k_{p}=\omega_{p} / c$ and timescales by the plasma frequency $\omega_{p}=\sqrt{4 \pi n_{0} e^{2} / m}$, where $c$ is the speed of light. Furthermore, densities are normalized to the ambient plasma electron density $n_{0}$, charges to the elementary charge $e$, masses to the electron mass $m$, and potentials to $m c^{2} / e$.

The parameters $c_{r}(\xi)$ and $c_{\psi}(\xi)$ in Eq. (1), introduced in Ref. 8 , account for the $\xi$-dependence of the blowout radius and the beam current, and for relativistic velocities of electrons in the sheath. The original hosing model for the adiabatically generated ion channel with non-relativistic sheath electrons is retrieved for $c_{r} c_{\psi}=1$. However, for $c_{r} c_{\psi}<1$ the growth rate is reduced. ${ }^{8}$

The coefficients $\lambda(\xi, t)$ and $\Omega(\xi, t)$ in Eq. (2) were introduced in Ref. 9. A finite $\lambda(\xi, t)$ accounts for the damping/ amplification of the beam centroid oscillations owing to a relativistic mass gain/loss of beam electrons as well as the damping of the centroid oscillations from a finite uncorrelated beam energy spread. The coefficient $\Omega(\xi, t)$ incorporates the effect of the change of the betatron frequency for a changing energy. If different slices of the beam gain or lose energy at differing rates, the beam centroid oscillations become detuned and hosing stops. ${ }^{9}$

The coefficients $c_{r}(\xi)$ and $c_{\psi}(\xi)$ in the plasma centroid equation (1) were derived in Ref. 8 through investigation 
of the dynamics of a plasma electron at the blowout-sheath boundary, so as to infer the perturbation of the channel centroid. This model posed a dramatic improvement in the modeling of hosing in PWFAs in the nonlinear blowout regime. However, as discussed within this work, this model is not sufficiently accurate, and in some regards, provides unphysical results. In this work, we therefore generalize the plasma centroid equation by investigating the collective dynamics of all sheath electrons subject to beam and plasma centroid deviations. As shown, the model introduced here is more accurate and can provide the basis for crucial studies of hosing of drive and witness beam pairs in PWFAs in the blowout regime.

The paper is organized as follows. In Sec. II, we derive a general moment equation and from this, we derive the plasma centroid equation. The plasma centroid equation is then applied to the blowout regime in Sec. III and compared to threedimensional (3D) particle-in-cell (PIC) simulation results with HiPACE $^{12}$ in Sec. IV. Section V concludes this work.

\section{DERIVATION OF THE PLASMA CENTROID EQUATION}

\section{A. Moment equation for the plasma electron phase space density}

In the following, we derive a general equation for the evolution of the moments of the plasma-electron phase space density $f_{p}$ along $\xi$, when the electrons are subject to the fields of a beam with a finite centroid displacement and to the fields of a blowout wake with a finite transverse displacement. We use the quasi-static approximation ${ }^{13-15}$ for the plasmaelectron phase space density, $\partial_{t} f_{p} \ll \partial_{\xi} f_{p}$. This approximation entails a constant of motion $\gamma_{e}-P_{z}-\Psi$ for plasma electrons. ${ }^{16}$ Here, $\gamma_{e}$ denotes the Lorentz factor of an electron, $P_{z}$ the longitudinal momentum of an electron, and $\Psi=\phi-A_{z}$ the wakefield potential at the instantaneous location of the electron, with $\phi$ being the scalar potential and $A_{z}$ the longitudinal component of the vector potential. Considering that electrons are initially at rest, the constant of motion allows for the introduction of the phase space variable $\psi=\gamma-p_{z}-1$ which is, via integration over all momentum phase space, associated with the wakefield potential $\Psi$. Here, $\gamma$ refers to the Lorentz factor as a function of the phase space momentum coordinates and $p_{z}$ denotes the longitudinal phase space momentum coordinate. Hence, for a given time $t$ and a comoving position $\xi$, the phase space distribution of the plasma electrons in an azimuthally symmetric plasma wave, $f_{p, 0}$, is a function only of radial position $r$, radial momentum $p_{r}$, and $\psi$, such that $f_{p, 0}=f_{p, 0}\left(r, p_{r}, \psi ; \xi, t\right)$.

From the conservation of the phase space density $d f_{p, 0} /$ $d t=0$, we obtain the Vlasov equation for $f_{p, 0}$ in the quasistatic approximation ${ }^{17}$

$$
\partial_{\xi} f_{p, 0}=-\frac{\gamma}{1+\psi}\left(\frac{p_{r}}{\gamma} \partial_{r}+F_{r} \partial_{p_{r}}+F_{\psi} \partial_{\psi}\right) f_{p, 0},
$$

with the forces $F_{r}=d p_{r} / d t$ and $F_{\psi}=d \psi / d t$, and where the Lorentz factor is expressed as

$$
\gamma=\frac{1+p_{r}^{2}+(1+\psi)^{2}}{2(1+\psi)} .
$$

When subject to hosing, the phase space distribution of the plasma electrons is not azimuthally symmetric. In leading order of a moment expansion of the phase space distribution within a slice, the perturbation owed to hosing results in a finite mean transverse position $\langle x\rangle$ and a finite mean transverse momentum $\left\langle p_{x}\right\rangle$. We therefore express the asymmetric plasma electron phase space distribution $f_{p}$ as an expansion of $f_{p, 0}\left(r^{*}, p_{r}^{*}, \psi ; \xi, t\right)$ for small $\langle x\rangle$ and $\left\langle p_{x}\right\rangle$, where $r^{*}=\sqrt{(x-\langle x\rangle)^{2}+y^{2}}$ and $p_{r}^{*}=\left(p_{x}-\left\langle p_{x}\right\rangle\right) \cos \theta+p_{y} \sin \theta$, such that

$$
f_{p} \simeq-\cos \theta\left(\langle x\rangle \partial_{r}+\left\langle p_{x}\right\rangle \partial_{p_{r}}\right) f_{p, 0},
$$

and $f_{p}=f_{p}\left(r, \theta, p_{r}, \psi ; \xi, t\right)$. The truncation of this expansion after the first order in $\langle x\rangle$ and $\left\langle p_{x}\right\rangle$ accurately reproduces the plasma electron phase space distribution in the initial phase of the hose instability before beam breakup occurs.

The Vlasov equation for the perturbed plasma electron distribution is

$$
\partial_{\xi} f_{p}=-\frac{\gamma}{1+\psi}\left(\frac{p_{r}}{\gamma} \partial_{r}+\dot{\theta} \partial_{\theta}+F_{r} \partial_{p_{r}}+F_{\psi} \partial_{\psi}\right) f_{p} .
$$

We are interested in moments of the phase space density, which are defined as

$$
\left\langle\Phi\left(r, \theta, p_{r}, \psi\right)\right\rangle(\xi, t)=\frac{1}{N} \int_{0}^{\infty} r d r \int_{0}^{2 \pi} d \theta \int_{-\infty}^{\infty} d p_{r} \int_{-1}^{\infty} d \psi \Phi f_{p},
$$

where the normalization is given by

$$
\begin{aligned}
N & =\int_{0}^{\infty} r d r \int_{0}^{2 \pi} d \theta \int_{-\infty}^{\infty} d p_{r} \int_{-1}^{\infty} d \psi f_{p} \\
& =2 \pi \int_{0}^{\infty} r d r \int_{-\infty}^{\infty} d p_{r} \int_{-1}^{\infty} d \psi f_{p, 0} .
\end{aligned}
$$

Moments with respect to the unperturbed phase space distribution are denoted by $\langle\Phi\rangle_{0}$ and are defined as

$$
\left\langle\Phi\left(r, p_{r}, \psi\right)\right\rangle_{0}(\xi, t)=\frac{2 \pi}{N} \int_{0}^{\infty} r d r \int_{-\infty}^{\infty} d p_{r} \int_{-1}^{\infty} d \psi \Phi f_{p, 0} .
$$

The multiplication of the Vlasov equation (6) with a quantity $\Phi$ and integration by parts with the assumption that the phase space density decays to zero at the integration limits yields the general moment equation

$$
\begin{aligned}
\partial_{\xi}\langle\Phi\rangle= & \left\langle\frac{p_{r}}{1+\psi} \partial_{r} \Phi\right\rangle+\left\langle\frac{\gamma \dot{\theta}}{1+\psi} \partial_{\theta} \Phi\right\rangle \\
& +\left\langle\frac{\gamma F_{r}}{1+\psi} \partial_{p_{r}} \Phi\right\rangle+\left\langle\frac{\gamma F_{\psi}}{1+\psi} \partial_{\psi} \Phi\right\rangle .
\end{aligned}
$$

This equation expresses how a macroscopic property $\langle\Phi\rangle$ of the plasma electron phase space distribution in a slice changes along the comoving coordinate. 


\section{B. Plasma centroid equation}

The plasma centroid in the context of the hose instability can be defined by the symmetry axis of the wakefield potential $\Psi$, towards which the highly relativistic beam electrons are attracted. For a cylindrically symmetric case, the wakefield exerts a force $\propto \partial_{r} \Psi_{0}$ on beam electrons towards the axis, where $\Psi_{0}=\Psi_{0}(r)$ is the cylindrically symmetric wakefield potential. We now expand the wakefield potential around a small centroid deviation $X_{p}$

$$
\Psi(r, \theta) \simeq-\cos \theta X_{p} \partial_{r} \Psi_{0}(r),
$$

such that the beam electrons are attracted towards the point $X_{p}$. In order to investigate how $X_{p}$ is connected with $\langle x\rangle$ in Eq. (5), we consider Maxwell equations in the quasi-static approximation using the Lorenz gauge (see, e.g., Ref. 5 or 18)

$$
\nabla_{\perp}^{2}\left[\begin{array}{c}
\Psi \\
A_{z}
\end{array}\right]=-\left[\begin{array}{c}
\rho-J_{z} \\
J_{z}
\end{array}\right],
$$

where $\rho$ is the charge density, $J_{z}$ the longitudinal current density, $A_{z}$ the longitudinal component of the vector potential, and $\Psi$ the wakefield potential, as defined before. The Lorenz gauge condition is given by ${ }^{18}$

$$
\nabla_{\perp} \cdot \mathbf{A}_{\perp}=-\frac{\partial \Psi}{\partial \xi} .
$$

Using Eqs. (11) and (12), the source term of the wakefield potential, $S=-\left(\rho-J_{z}\right)$, is

$$
S(r, \theta) \simeq-\cos \theta X_{p} \partial_{r} S_{0}(r)
$$

for $r \gg\left|X_{p}\right|$, where $S_{0}=S_{0}(r)$ refers to the cylindrically symmetric charge and current distribution, which gives rise to the symmetric wakefield potential according to $\nabla_{\perp}^{2} \Psi_{0}$ $=S_{0}$. The source term $S$ is given by the plasma charge and current distribution and connected to the plasma phase space distribution via

$$
S=-1+\int_{-\infty}^{\infty} d p_{r} \int_{-1}^{\infty} d \psi \frac{1+\psi}{\gamma} f_{p}
$$

where the first term corresponds to the background ion contribution and where the identity $1-p_{z} / \gamma=(1+\psi) / \gamma$ was used. Inserting the phase space expansion according to Eq. (5) in Eq. (15) and performing integration by parts show that the wakefield centroid $X_{p}$ is equivalent to the plasma electron centroid $\langle x\rangle$ when assuming that the transverse momentum deviation $\left\langle p_{x}\right\rangle$ does not cause a significant relativistic mass change of plasma electrons, i.e., if $\left\langle p_{x}\right\rangle \partial_{p_{r}} \gamma \approx 0$.

Hence, in the following, we investigate the evolution of the plasma centroid $\langle x\rangle \simeq X_{p}$ along the co-moving variable $\xi$ for small perturbations $\langle x\rangle$ and $\left\langle p_{x}\right\rangle$. Using the moment equation (10), the change of the plasma centroid with respect to the co-moving variable is given by

$$
\partial_{\xi}\langle x\rangle=\partial_{\xi}\langle r \cos \theta\rangle=\left\langle\frac{p_{r} \cos \theta}{1+\psi}\right\rangle .
$$

The moment term containing $\partial_{\theta}(r \cos \theta)$ vanishes. Expanding $1 /(1+\psi)$ around $\langle\psi\rangle$ yields

$$
\left\langle\frac{p_{r} \cos \theta}{1+\psi}\right\rangle=\left\langle\frac{p_{r} \cos \theta}{\langle 1+\psi\rangle} \sum_{k=0}^{\infty}(-1)^{k}\left(\frac{\psi-\langle\psi\rangle}{\langle 1+\psi\rangle}\right)^{k}\right\rangle .
$$

In the linear regime, $|\psi| \simeq\left|p_{z}\right| \ll 1$, the above series expansion converges to

$$
\left\langle\frac{p_{r} \cos \theta}{1+\psi}\right\rangle \simeq \frac{\left\langle p_{r} \cos \theta\right\rangle}{\langle 1+\psi\rangle}
$$

This is also a valid approximation for the blowout regime as discussed in Appendix A. Using the moment equation (10), the change of the electron-plasma centroid along the comoving variable is then given by

$$
\begin{aligned}
\partial_{\xi}^{2}\langle x\rangle & =\frac{\left\langle\frac{\gamma}{1+\psi} F_{r} \cos \theta\right\rangle}{\langle 1+\psi\rangle}-\frac{\left\langle p_{r} \cos \theta\right\rangle}{\langle 1+\psi\rangle^{2}}\left\langle\frac{\gamma F_{\psi}}{1+\psi}\right\rangle \\
& =\frac{\left\langle\frac{\gamma}{1+\psi} F_{r} \cos \theta\right\rangle}{\langle 1+\psi\rangle}-\frac{\partial_{\xi}\langle x\rangle}{\langle 1+\psi\rangle}\left\langle\frac{\gamma F_{\psi}}{1+\psi}\right\rangle .
\end{aligned}
$$

The radial force $F_{r}$ acting on the plasma electrons is composed of the force exerted by the fields originating from the perturbed plasma charge and current densities $F_{r, p}(r, \theta, \xi)$, and a force exerted by the fields originating from the beam charge and longitudinal current densities $F_{r, b}(r, \theta, \xi)$, such that $F_{r}=F_{r, p}+F_{r, b}$. These forces are expanded as

$$
\begin{aligned}
& F_{r, b} \simeq-\cos \theta X_{b} \partial_{r} F_{r, b, 0}, \\
& F_{r, p} \simeq-\cos \theta\langle x\rangle \partial_{r} F_{r, p, 0},
\end{aligned}
$$

where $F_{r, p, 0}(r, \xi)$ and $F_{r, b, 0}(r, \xi)$ refer to the respective unperturbed, azimuthally symmetric forces.

We now use the expansions (5), (20a), and (20b) together with $F_{r}=F_{r, p}+F_{r, b}$ in the plasma centroid equation (19). Truncation after $\mathcal{O}\left(X_{b}\right), \mathcal{O}(\langle x\rangle)$, and $\mathcal{O}\left(\left\langle p_{x}\right\rangle\right)$ yields

$$
\begin{aligned}
\partial_{\xi}^{2}\langle x\rangle= & -\frac{\langle x\rangle}{2\langle 1+\psi\rangle_{0}}\left\langle\frac{\gamma}{1+\psi} \partial_{r} F_{r, p, 0}\right\rangle_{0} \\
& -\frac{X_{b}}{2\langle 1+\psi\rangle_{0}}\left\langle\frac{\gamma}{1+\psi} \partial_{r} F_{r, b, 0}\right\rangle_{0} \\
& +\frac{\langle x\rangle}{2\langle 1+\psi\rangle_{0}}\left\langle\frac{\gamma}{1+\psi}\left(\partial_{r}+r^{-1}\right) F_{r, p, 0}\right\rangle_{0} \\
& +\frac{\langle x\rangle}{2\langle 1+\psi\rangle_{0}}\left\langle\frac{\gamma}{1+\psi}\left(\partial_{r}+r^{-1}\right) F_{r, b, 0}\right\rangle_{0} \\
& +\partial_{\xi}\langle x\rangle\left\langle\frac{p_{r} F_{r, 0}}{(1+\psi)^{2}}\right\rangle_{0} \\
& -\frac{\partial_{\xi}\langle x\rangle}{\langle 1+\psi\rangle_{0}}\left\langle\frac{\gamma F_{\psi}}{1+\psi}\right\rangle_{0},
\end{aligned}
$$

where integration by parts was used to obtain the third, fourth, and fifth terms and where the identities $\int_{0}^{2 \pi} \cos ^{2} \theta f_{p, 0} d \theta$ $=\pi f_{p, 0}$ and $\left\langle p_{x}\right\rangle / 2=\left\langle p_{r} \cos \theta\right\rangle=\langle 1+\psi\rangle \partial_{\xi}\langle x\rangle$ were used. 
Note that all moments, apart from $\langle x\rangle$, are now defined in terms of the unperturbed phase space density $f_{p, 0}$. From this point on, $\langle x\rangle$ is denoted by $X_{p}$, and Eq. (21) is rearranged to form of the second-order differential plasma centroid equation

$$
\frac{\partial^{2} X_{p}}{\partial \xi^{2}}+\mathcal{C}_{d}(\xi) \frac{\partial X_{p}}{\partial \xi}+\frac{\mathcal{C}_{p}(\xi)}{2} X_{p}=\frac{\mathcal{C}_{b}(\xi)}{2} X_{b} .
$$

This equation is equivalent to a driven, damped harmonic oscillator for the plasma centroid $X_{p}(\xi, t)$ along $\xi$. The second term thereby acts as a damping/amplifying term for $\mathcal{C}_{d} \neq 0$ and the coefficient $\mathcal{C}_{p} / 2$ is the square of the undamped oscillation wavenumber of the system. The oscillator is driven by the beam centroid $X_{b}$ for a finite $\mathcal{C}_{b}$. The coefficients $\mathcal{C}_{b}(\xi), \mathcal{C}_{p}(\xi)$ and $\mathcal{C}_{d}(\xi)$ are given by the respective moments in Eq. (21)

$$
\begin{gathered}
\mathcal{C}_{p}=-\frac{1}{\langle 1+\psi\rangle_{0}}\left(\left\langle\frac{\gamma}{1+\psi} r^{-1} F_{r, p, 0}\right\rangle_{0}\right. \\
\left.+\left\langle\frac{\gamma}{1+\psi}\left(\partial_{r}+r^{-1}\right) F_{r, b, 0}\right\rangle_{0}\right), \\
\mathcal{C}_{b}=-\frac{1}{\langle 1+\psi\rangle_{0}}\left\langle\frac{\gamma}{1+\psi} \partial_{r} F_{r, b, 0}\right\rangle_{0}, \\
\mathcal{C}_{d}=\frac{1}{\langle 1+\psi\rangle_{0}}\left\langle\frac{\gamma F_{\psi}}{1+\psi}\right\rangle_{0}-\left\langle\frac{p_{r} F_{r, 0}}{(1+\psi)^{2}}\right\rangle_{0} .
\end{gathered}
$$

For the calculation of $\mathcal{C}_{p}$, we used the fact that the first term in Eq. (21) cancels the part of the third term which includes the radial derivative of $F_{r, p, 0}$. The coefficient $\mathcal{C}_{b}$ determines the amount a local beam centroid deviation contributes to the curvature of the plasma centroid along $\xi$. The respective contributions from the plasma centroid and the slope of the plasma centroid are incorporated by the coefficients $\mathcal{C}_{p}$ and $\mathcal{C}_{d}$, respectively. A nonzero $\mathcal{C}_{d}$ results in a damping/amplification owing to relativistic mass gain/loss of plasma electrons along the plasma wave. To simplify $\mathcal{C}_{d}$, we apply the Lorentz force to $\psi=\gamma-p_{z}-1$ to find that

$$
\frac{\gamma F_{\psi}}{1+\psi}=\frac{p_{r} F_{r, 0}}{1+\psi}-F_{z, 0} .
$$

Hence, expanding the second term in Eq. (23c) in a similar way to that in Eq. (18) gives

$$
\begin{aligned}
\mathcal{C}_{d} & =\frac{1}{\langle 1+\psi\rangle_{0}}\left\langle\frac{p_{r} F_{r, 0}}{1+\psi}\right\rangle-\left\langle\frac{p_{r} F_{r, 0}}{(1+\psi)^{2}}\right\rangle_{0}-\frac{\left\langle F_{z, 0}\right\rangle_{0}}{\langle 1+\psi\rangle_{0}} \\
& \simeq-\frac{\left\langle F_{z, 0}\right\rangle_{0}}{\langle 1+\psi\rangle_{0}} .
\end{aligned}
$$

The forces can be expressed in terms of electromagnetic potentials

$$
\begin{gathered}
F_{r, b, 0}=\frac{1+\psi}{\gamma} \partial_{r} A_{z, b}=-\frac{1+\psi}{\gamma} \frac{1}{r} \int_{0}^{r} r^{\prime} J_{z, b}\left(r^{\prime}\right) d r^{\prime}, \\
F_{r, p, 0}=\partial_{r} \Psi+\frac{1+\psi}{\gamma}\left(\partial_{\xi} A_{r}+\partial_{r} A_{z, p}\right), \\
F_{z, 0}=-\partial_{\xi} \Psi,
\end{gathered}
$$

where $A_{r}$ is the radial component of the vector potential. From here on, all potentials refer to the azimuthally symmetric potentials and the zero-subscripts are dropped for the potentials. Note that the beam only gives rise to the longitudinal wakefield potential $A_{z, b}$. The wakefield potential $\Psi$ and radial vector potential $A_{r}$ are generated by the plasma charge and current densities.

Using Eq. (26a), the radial derivative of $F_{r, b, 0}$ can be written as

$$
\partial_{r} F_{r, b, 0}(r)=-\frac{1+\psi}{\gamma}\left[\frac{\partial_{r} A_{z, b}(r)}{r}+J_{z, b}(r)\right] .
$$

Expressing the coefficients $\mathcal{C}_{p}, \mathcal{C}_{b}$, and $\mathcal{C}_{d}$ in terms of the potentials therefore gives

$$
\begin{gathered}
\mathcal{C}_{p}=-\frac{1}{\langle 1+\psi\rangle_{0}}\left(\left\langle\frac{\gamma}{1+\psi} \frac{\partial_{r} \Psi}{r}\right\rangle_{0}+\left\langle\frac{\partial_{\xi} A_{r}}{r}\right\rangle_{0}\right. \\
\left.+\left\langle\frac{\partial_{r} A_{z, p}}{r}\right\rangle_{0}-\left\langle J_{z, b}\right\rangle_{0}\right), \\
\mathcal{C}_{b}=\frac{1}{\langle 1+\psi\rangle_{0}}\left\langle\frac{\partial_{r} A_{z, b}}{r}+J_{z, b}\right\rangle_{0}, \\
\mathcal{C}_{d}=\frac{\left\langle\partial_{\xi} \Psi\right\rangle_{0}}{\langle 1+\psi\rangle_{0}} .
\end{gathered}
$$

In the following, we assume that at a given radial position, the phase space distribution $f_{p, 0}\left(r, p_{r}, \psi\right)$ features a cold fluid distribution, such that

$$
f_{p, 0}\left(r, p_{r}, \psi\right)=\tilde{n}(r) \delta\left(p_{r}-P_{r}(r)\right) \delta(\psi-\Psi(r)),
$$

where $\tilde{n}(r)=n(r)-n_{0}$ is the excess electron density. Note that this cold fluid assumption is in good agreement with observations from PIC simulations for regions ahead of the blowout vertex (electron density spike) as shown in previous works. $^{18-20}$

In the following, we express the averaged quantities in Eqs. (28a), (28b), and (28c) as functions of the radius $r$ and comoving position $\xi$. This can be done by use of Eq. (29) which allows to form fluid or momentum moments such as

$$
\begin{aligned}
\iint \frac{\gamma}{1+\psi} f_{p, 0} d \psi d p_{r} & =\iint \frac{\gamma}{\gamma-p_{z}} f_{p, 0} d \psi d p_{r} \\
& =\frac{\tilde{n}}{\tilde{n}-J_{z, p}}=1+\frac{J_{z, p}}{\tilde{n}-J_{z, p}} .
\end{aligned}
$$

The coefficients $\mathcal{C}_{b}, \mathcal{C}_{p}$, and $\mathcal{C}_{d}$ can now be fully expressed by radial moments of potentials and charge and current densities

$$
\begin{gathered}
\mathcal{C}_{p}=-\frac{1}{\langle 1+\Psi\rangle_{r}}\left[\left\langle\left(1+\frac{J_{z, p}}{\tilde{n}-J_{z, p}}\right) \frac{\partial_{r} \Psi}{r}\right\rangle_{r}+\left\langle\frac{\partial_{\xi} A_{r}}{r}\right\rangle_{r}\right. \\
\left.+\left\langle\frac{\partial_{r} A_{z, p}}{r}\right\rangle_{r}-\left\langle J_{z, b}\right\rangle_{r}\right] \\
\mathcal{C}_{b}=\frac{1}{\langle 1+\Psi\rangle_{r}}\left[\left\langle\frac{\partial_{r} A_{z, b}}{r}\right\rangle_{r}+\left\langle J_{z, b}\right\rangle_{r}\right]
\end{gathered}
$$




$$
\mathcal{C}_{d}=\frac{\left\langle\partial_{\xi} \Psi\right\rangle_{r}}{\langle 1+\Psi\rangle_{r}}
$$

where the radial moments are defined by

$$
\langle\Phi(r)\rangle_{r}=\frac{1}{N} \int_{0}^{\infty} \tilde{n}(r) \Phi(r) r d r .
$$

Hence, if current densities and potentials are known for the azimuthally symmetric case, the above coefficients can be computed, allowing for the investigation of the plasma centroid evolution along the beam by means of Eq. (22).

An electron beam implies $\mathcal{C}_{b} \geq 0$, such that a positive beam centroid deviation $X_{b}$ exerts a "kick" in the positive $x$ direction onto the plasma centroid $X_{p}$. For $\mathcal{C}_{p}>0$, the plasma centroid is restored by the self-consistent plasma electron and ion fields; however, if $\mathcal{C}_{p}<0$, the selfconsistent plasma and ion fields enhance the plasma centroid deviation $X_{p}$. The term in Eq. (22) results in a damping of the plasma centroid for $\mathcal{C}_{d}>0$, while the plasma centroid is amplified for $\mathcal{C}_{d}<0$.

\section{BLOWOUT REGIME AND NARROW BEAMS}

\section{A. Plasma wake centroid equation}

As an example, we consider the nonlinear blowout regime. ${ }^{7}$ In Ref. 8, the evolution of the blowout centroid subject to a narrow beam with asymmetric charge distribution was derived by studying the perturbed dynamics of a plasma electron at the boundary of the blowout and sheath. In the present work, we study the collective response of the electron sheath to beam centroid and plasma centroid displacements and show that by considering a finite sheath thickness, we obtain a different blowout channel equation with different and/or reduced coupling coefficients.

In this context, "narrow beam" refers to the case when the overlap of beam current density and electron sheath is negligible, $\left\langle J_{z, b}\right\rangle_{r} \simeq 0$. Hence, for the narrow beam case, the coefficients (31a), (31b), and (31c) simplify to

$$
\begin{gathered}
\mathcal{C}_{p}=-\frac{1}{\langle 1+\Psi\rangle_{r}}\left[\left\langle\left(1+\frac{J_{z, p}}{\tilde{n}-J_{z, p}}\right) \frac{\partial_{r} \Psi}{r}\right\rangle_{r}\right. \\
\left.+\left\langle\frac{\partial_{\xi} A_{r}}{r}\right\rangle_{r}+\left\langle\frac{\partial_{r} A_{z, p}}{r}\right\rangle_{r}\right], \\
\mathcal{C}_{b}=\frac{1}{\langle 1+\Psi\rangle_{r}}\left[\left\langle\frac{\partial_{r} A_{z, b}}{r}\right\rangle_{r}\right], \\
\mathcal{C}_{d}=\frac{\left\langle\partial_{\xi} \Psi\right\rangle_{r}}{\langle 1+\Psi\rangle_{r}} .
\end{gathered}
$$

The explicit computation of the radial moments in (33a), (33b), and (33c) requires the knowledge of the sheath current and charge density distributions and the potentials within the sheath. For this purpose we employ the blowout model in Ref. 18 in which the spatial distribution of the sheath plasma electron density is assumed to be given by

$$
\tilde{n}=\tilde{n}_{s} \exp \left(-\frac{r-R}{\Delta_{\rho}}\right) \Theta(r-R),
$$

with the blowout radius $R=R(\xi)$, the sheath thickness $\Delta_{\rho}$, the peak sheath electron density $\tilde{n}_{s}=\tilde{n}_{s}(\xi)$, and $\Theta(x)$ is the Heaviside step function (compare illustration in Fig. 1). Following Ref. 18, we also assume that the quantity $S$ $=-\left(\tilde{n}-J_{z, p}\right)$ follows the same radial dependence as $\tilde{n}$.

The plasma electron current is assumed to be given by the functional dependence

$$
J_{z, p}=J_{s} \exp \left(-\frac{r-R}{\Delta_{J}}\right) \Theta(r-R),
$$

where $J_{s}=J_{s}(\xi)$ is the peak return current and $\Delta_{J}$ is the current layer thickness. We use the results obtained in Ref. 18 for the electromagnetic potentials $\Psi(\xi, r), A_{r}(\xi, r), A_{z, b}(\xi, r)$, and $A_{z, p}(\xi, r)$, with the approximation that the normalized sheath thickness is much smaller than the local blowout radius $\Delta_{\rho} /$ $R \ll 1$. This thin sheath approximation is consistent with results from PIC simulations (see, e.g., Refs. 19 and 20). The plasma return-current layer thickness is assumed to spread over a plasma skin depth, $\Delta_{J} \simeq 1$, as assumed in Ref. 18 .

Using these assumptions, the coefficient (33a), which determines the magnitude of the plasma response to a plasma centroid deviation in Eq. (22), yields

$$
\begin{aligned}
\mathcal{C}_{p}= & \frac{1-R^{\prime 2}}{4}-\frac{\Delta_{\rho}}{4}\left[\frac{1+\Lambda}{R}+\frac{R\left(R^{\prime 2}-R^{2}-1\right)}{4}-\frac{R^{2} R^{\prime}}{2}\right] \\
& +\mathcal{O}\left(\frac{\Delta_{\rho}^{2}}{R^{2}}\right)
\end{aligned}
$$

where $R^{\prime}(\xi)=\partial_{\xi} R(\xi)$ is the slope of the blowout radius and $\Lambda(\xi)=4 I_{b} / I_{A}$ is the normalized beam current, where the beam current is denoted by $I_{b}(\xi)$ and the Alfvén current by $I_{A} \simeq 17 \mathrm{kA}$. Equation (36) implies a restoring effect of the plasma wakefields onto the plasma centroid if $R^{\prime 2} \lesssim 1$ and an amplifying effect if $R^{\prime 2}>1$. The latter can be explained by the fact that the wakefield exerts a force in the positive $r$-direction onto sheath electrons at the rear of the blowout (see, for example, Refs. 21 and 22). Hence, at the rear of the

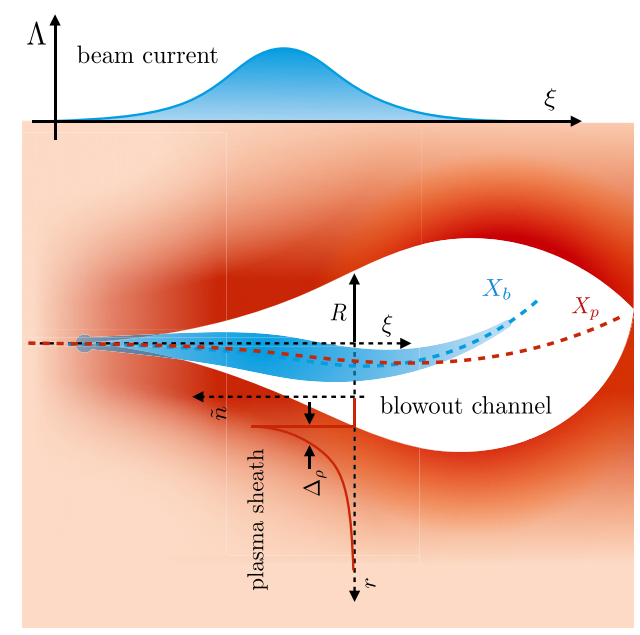

FIG. 1. Illustration of a blowout channel and the relevant beam and plasma quantities. 
blowout, where $R^{\prime 2}$ is large, wakefield perturbations may be enhanced towards the vertex of the blowout.

The coefficient (33b), defining the magnitude of the expelling force exerted onto the plasma centroid by the beam centroid, becomes

$$
\mathcal{C}_{b}=\frac{\Lambda}{R^{2}}\left[1-\Delta_{\rho}\left(\frac{2}{R}+\frac{R}{4}\right)\right]+\mathcal{O}\left(\frac{\Delta_{\rho}^{2}}{R^{2}}\right) .
$$

The centroid deviation of an electron beam acts as a force onto the plasma centroid, where the magnitude of the effect scales with the beam current and inversely with the square of the blowout radius. A finite sheath thickness $\Delta_{\rho}$ reduces this effect. Analogously, the damping-coefficient (33c) reads

$$
\mathcal{C}_{d}=\frac{R^{\prime}}{4}\left[R-\frac{\Delta_{\rho}}{2}\left(\frac{R^{2}}{2}-1\right)\right]+\mathcal{O}\left(\frac{\Delta_{\rho}^{2}}{R^{2}}\right) .
$$

The coefficient $\mathcal{C}_{d}$ therefore implies a damping of the plasma centroid due to a relativistic mass gain of electrons in the sheath if $R^{\prime}>0$ and an amplification owing to a relativistic mass loss for $R^{\prime}<0$.

\section{B. Discussion and comparison to previous work}

We compare these results to the plasma centroid equations obtained in previous works. In the seminal work on the hose instability in plasma wakefield accelerators, Whittum et al. ${ }^{5}$ assumed an adiabatically generated ion channel at the charge neutralization radius and with non-relativistic sheath electrons. Hence, the channel centroid equation used by Whittum et al. corresponds to Eq. (22) with $\mathcal{C}_{p}=\mathcal{C}_{b}=1$ and $\mathcal{C}_{d}=0$.

In a more recent work, it was shown that this assumption greatly overestimates the growth rate of the hose instability in the nonlinear blowout regime. ${ }^{8}$ In Ref. 8, the plasma centroid equation was generalized by investigation of the dynamics of an electron at the blowout-sheath boundary so as to furnish a hosing model which includes the effects of a varying beam current, changing blowout radius, and relativistic velocities of electrons in the sheath. The channel centroid equation in Ref. 8 is given by Eq. (1), where $c_{r}=\Lambda / R^{2}$ and $c_{\psi}=[1+\Psi(R)]^{-1}$. This is equivalent to Eq. (22) with coefficients $\mathcal{C}_{p}=\mathcal{C}_{b}=c_{r} c_{\psi} \simeq \Lambda / R^{2}\left(1-\Delta_{\rho} R / 2\right)+\mathcal{O}\left(\Delta_{\rho}^{2} / R^{2}\right)$ and $\mathcal{C}_{d}=0$.

In the limit $\Delta_{\rho} \rightarrow 0$, the model in Ref. 8 and the coefficient $\mathcal{C}_{b}$ derived in this work, Eq. (37), recover the same result $\mathcal{C}_{b} \rightarrow \Lambda / R^{2}$. That is, for $\Delta_{\rho}=0$, the impact of the beam centroid deviation onto the plasma centroid deviation scales linearly with the beam current and inversely with the square of the blowout radius. However, for a finite sheath thickness, $\Delta_{\rho}>0$ we find a reduced coefficient $\mathcal{C}_{b}$ for blowout radii $R \sim 1$. This is shown in Fig. 2, which depicts a comparison of $\mathcal{C}_{b} / \Lambda$ for varying blowout radii as predicted by the model derived within the present work and from the model in Ref. 8. It can be seen that the model derived within this work predicts a smaller value of $\mathcal{C}_{b} / \Lambda$ for moderate radii and finite $\Delta_{\rho}$.

In addition, and in contrast to previous models ${ }^{5,8}$ we find a coefficient corresponding to the restoring force of the plasma centroid which differs from the coefficient for the driving

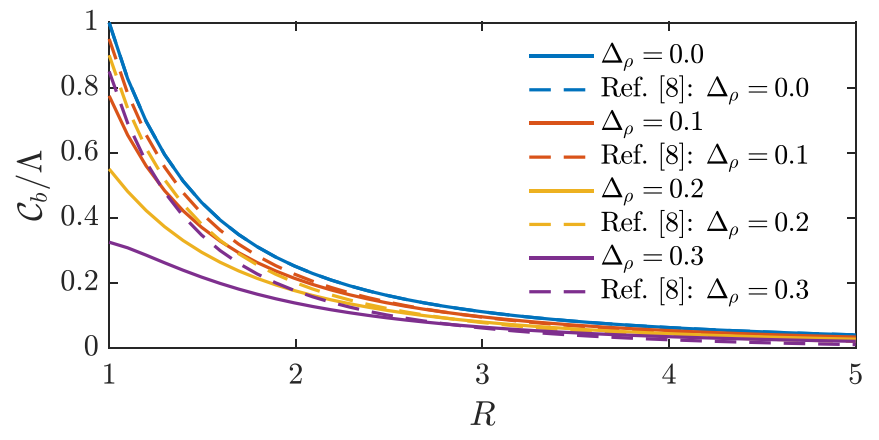

FIG. 2. Depiction of the coefficient $\mathcal{C}_{b}$, relative to the beam current $\Lambda$ as a function of the blowout radius $R$ for differing sheath thicknesses $\Delta_{\rho}$. Solid lines refer to $\mathcal{C}_{b}$ derived within the present work and dashed lines to $\mathcal{C}_{b}\left(=\mathcal{C}_{p}\right)$ in Ref. 8.

force $\left(\mathcal{C}_{p} \neq \mathcal{C}_{b}\right)$. It should be noted that the plasma centroid equation (1) used in previous models ${ }^{5,8}$ has the unphysical property that $\partial_{\xi} X_{p}=$ const if the beam current drops to zero $\left(\Lambda=0, c_{r} c_{\psi}=0\right)$. This means that previous models predict that the plasma centroid to maintain the slope it has at the beam tail. In contrast, for the model derived within this work, $\mathcal{C}_{p} \neq 0$ for $\Lambda=0$. Hence, the plasma centroid in this model experiences a restoring force and oscillates after the beam passed according to Eq. (22). The correct reproduction of this physical behavior is especially important for the study of hosing of a driver-witness pair, where the witness beam is subject to the plasma centroid deviations generated by the drive beam.

Furthermore, our model includes the effect of damping or amplification of the channel centroid deviation $\left(\mathcal{C}_{d} \neq 0\right)$ owing to a relativistic mass gain or loss of the sheath electrons as they propagate along the blowout. This effect is expected to be significant at the rear of the blowout where $E_{z}$ and the slope of the blowout radius can be substantial. Hence, a nonzero damping can be of particular relevance for the study of hosing of a witness beam which might be positioned near the end of the blowout cavity.

\section{COMPARISON TO SIMULATION RESULTS}

In this section, we compare the new model to results from 3D PIC simulations using the code HiPACE. ${ }^{12} \mathrm{We}$ investigate the hosing of a drive beam with a triangular current profile. The beam parameters are the same as the ones used in Ref. 8. The beam has an energy of $\gamma_{0}=55773$, and a transverse Gaussian density distribution with transverse dimensions $\sigma_{x}=\sigma_{y}=13.0 \times 10^{-3}$. The current profile is triangular with the current rising from zero at $\xi=-0.8$ to the peak value of $\hat{I}_{b}=6.65 \mathrm{kA}$ at $\xi=-0.53$. From this position, the current decreases linearly to zero at $\xi=3.19$. The initial beam centroid is given by $X_{b, 0}(\xi)=4.17 \times 10^{-3} \times \xi \Theta(\xi)$, such that a linear tilt is introduced for the beam from position $\xi=0$. The beam propagates through a flat-top plasma target with density $n_{0}$ and drives a plasma wave in the blowout regime.

We investigate how the channel/plasma centroid evolves along $\xi$ if excited by the initial centroid deviation of the beam. In order to be less dependent on the model for the blowout used, we evaluate the blowout radius $R(\xi)$ from the 


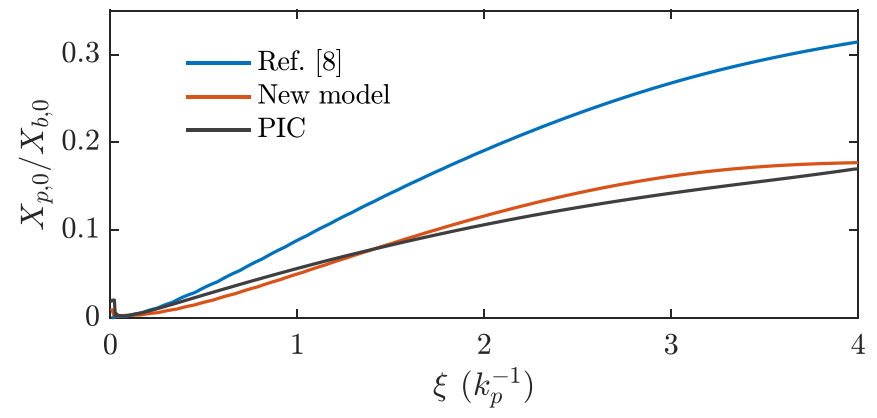

FIG. 3. Plasma centroid deviation along $\xi$ relative to initial beam centroid deviation at $t=0$ with $\Delta_{\rho}=0.2$.

PIC simulation. The blowout radius $R(\xi)$ and the change of the blowout radius $R^{\prime}(\xi)$ are then used to compute the coefficients $\mathcal{C}_{p}, \mathcal{C}_{b}$, and $\mathcal{C}_{d}$ in the present model and the coefficients $c_{r}$ and $c_{\psi}$ in the model from Ref. 8. We then solve numerically Eq. (22) and compare this to the numerical solution of Eq. (1) of the model in Ref. 8. The structure of the sheath in the simulation is compatible with Eq. (34) with $\Delta_{\rho}=0.2$. The results for $X_{p, 0} / X_{b, 0}$, where $X_{p, 0}=X_{p}(\xi, t=0)$ and $X_{b, 0}=X_{b}(\xi, t=0)$, are depicted in Fig. 3. The relative plasma centroid deviation obtained from the PIC simulation by interpolation to the zero crossing of the transverse fields $E_{x}-B_{y}$ is also shown. It can be seen that the new model (red line) is in good qualitative agreement with the PIC result (gray line), while the previous model (blue line) overestimates the channel centroid deviation.

In addition, the temporal beam centroid evolution is investigated. The beam is defined by the parameters above and the centroid is probed at the tail $(\xi=3)$. We numerically evaluate the model in Refs. 8 and 9 with the coupled differential equations for the plasma centroid, ${ }^{8}$ Eq. (1), and the beam centroid, ${ }^{9}$ Eq. (2). For the new model, we accordingly evaluate the new channel centroid equation (22) and the beam centroid equation (2) from Ref. 9. Furthermore, the longitudinal field $E_{z}(\xi)$ is extracted from the PIC simulation in order to avoid dependencies on the used blowout model.

Figure 4 depicts the comparison of the beam centroid at $k_{p} \xi=3$ as resulting from the model in Refs. 8 and 9 (blue line) and from the model for the plasma centroid derived within this work using the beam centroid from Ref. 9 (red line). Additionally, the beam centroid obtained from a PIC

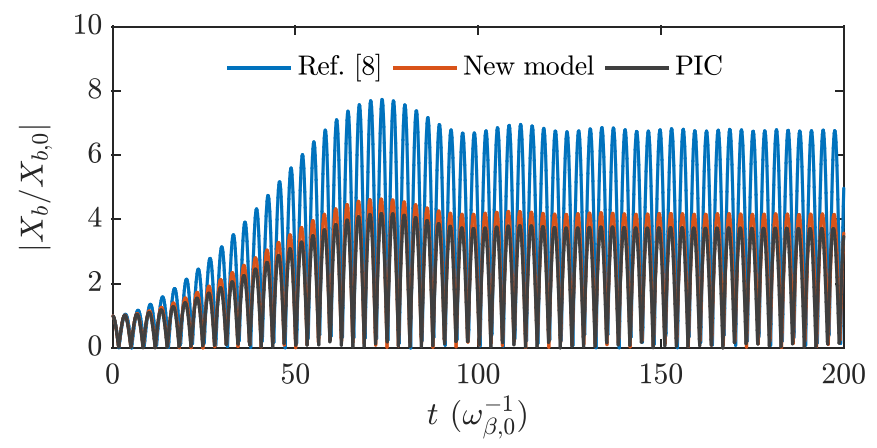

FIG. 4. Beam centroid evolution in time (normalized to the initial betatron frequency of the beam, $\left.\omega_{\beta, 0}\right)$ at the tail $(\xi=3)$. For the curves obtained from the models, $\Delta_{\rho}=0.2$ is used in agreement with the sheath thickness observed in the PIC simulation. simulation with HiPACE is shown (gray line). It can be seen that the beam centroid evolution expected by the present model is in good agreement with the PIC result, while the previous model overestimates the beam centroid deviation.

\section{CONCLUSION}

In this work, a generalized plasma centroid equation for the description of hosing in PWFAs in the blowout regime is derived. The differential equation for the plasma centroid is derived starting from the Vlasov equation and by considering the collective dynamics of the plasma electron sheath. This approach yields a mathematical model that predicts a reduced coupling between beam and plasma sheath and hence, accordingly reduced hosing growth rates for a finite sheath thickness compared to previous models. We also obtain a term associated with the damping/amplification of the plasma centroid, originating from the relativistic mass gain/loss of electrons in the sheath. Furthermore, a different coefficient for the restoring force of the plasma centroid is obtained which accounts for the fact that the plasma centroid is oscillating after the beam has passed. The inclusion of these effects yields results that are in better agreement with PIC simulations. The presented model is more accurate and general in the sense that it allows for the study of hosing of drive and witness beam pairs in PWFAs in the blowout regime, which was not possible with previous models. Hence, the presented model provides an important basis for the accurate modeling of hosing in PWFAs and for the possibility to study the stability of compact plasma-based accelerators.

\section{ACKNOWLEDGMENTS}

T.J.M. would like to thank R. E. Robson and J. Vieira for fruitful discussions. This work was partially funded through the Helmholtz Virtual Institute VH-VI-503 and Matter and Technologies ARD program. Simulations were performed on the Maxwell-Cluster at DESY. We acknowledge the support by the Director, Office of Science, Office of High Energy Physics, of the U.S. Department of Energy under Contract No. DE-AC02-05CH11231.

\section{APPENDIX A: CONVERGENCE OF THE EXPANSION FOR THE BLOWOUT REGIME}

In this Appendix, we evaluate Eq. (17) in Sec. II B

$$
\begin{aligned}
\left\langle\frac{p_{r} \cos \theta}{1+\psi}\right\rangle & =\left\langle\frac{p_{r} \cos \theta}{\langle 1+\psi\rangle} \sum_{k=0}^{\infty}(-1)^{k}\left(\frac{\psi-\langle\psi\rangle}{\langle 1+\psi\rangle}\right)^{k}\right\rangle \\
= & \left\langle\frac{p_{r} \cos \theta-\left\langle p_{r} \cos \theta\right\rangle}{\langle 1+\psi\rangle} \sum_{k=0}^{\infty}(-1)^{k}\left(\frac{\psi-\langle\psi\rangle}{\langle 1+\psi\rangle}\right)^{k}\right\rangle \\
& +\frac{\left\langle p_{r} \cos \theta\right\rangle}{\langle 1+\psi\rangle}\left\langle\sum_{k=0}^{\infty}(-1)^{k}\left(\frac{\psi-\langle\psi\rangle}{\langle 1+\psi\rangle}\right)^{k}\right\rangle, \quad \text { (A1) }
\end{aligned}
$$

for the blowout regime. Applying the expansion (5) to the covariance term (second line) of Eq. (A1) shows that this term vanishes by construction of $f_{p}$ as an expansion with respect to $f_{p, 0}$. We then note that $\langle\psi\rangle$ is dominated by the large fraction of electrons near the peak of the sheath density 
where the density decay along the radial position is much greater than the decay of the wakefield potential $\left|\partial_{r} n / n\right|$ $\gg\left|\partial_{r} \Psi / \Psi\right|$. Hence, $|\langle\psi\rangle| \lesssim|\Psi(R)| \sim \mathcal{O}\left(\Delta_{\rho} R\right)$ [compare $\Psi(R)$ computed in Ref. 18], where $\Delta_{\rho} \sim 0.1$ is the sheath thickness and $R \sim 1$ is the blowout radius. The first $(k=1)$ central moment is zero and the higher order central moments $(k>1)$ scale as $\left|\left\langle(\psi-\langle\psi\rangle)^{k}\right\rangle\right| \sim \mathcal{O}\left[\left(\Delta_{\rho} R\right)^{k}\right] \ll 1$. This is consistent with observations from PIC simulations. The second term (last line) of Eq. (A1) converges as

$$
\begin{gathered}
\frac{\left\langle p_{r} \cos \theta\right\rangle}{\langle 1+\psi\rangle}\left\langle\sum_{k=0}^{\infty}(-1)^{k}\left(\frac{\psi-\langle\psi\rangle}{\langle 1+\psi\rangle}\right)^{k}\right\rangle \\
=\frac{\left\langle p_{r} \cos \theta\right\rangle}{\langle 1+\psi\rangle}+\mathcal{O}\left[\left(\Delta_{\rho} R\right)^{2}\right] .
\end{gathered}
$$

Hence, the series expansion in Sec. II B can be approximated as

$$
\left\langle\frac{p_{r} \cos \theta}{1+\psi}\right\rangle \simeq \frac{\left\langle p_{r} \cos \theta\right\rangle}{\langle 1+\psi\rangle} .
$$

${ }^{1}$ V. I. Veksler, in Proceedings of the CERN Symposium on High-Energy Accelerators and Pion Physics (1956), p. 80.

${ }^{2}$ P. Chen, J. M. Dawson, R. W. Huff, and T. Katsouleas, Phys. Rev. Lett. 54, 693 (1985).

${ }^{3}$ I. Blumenfeld, C. E. Clayton, F.-J. Decker, M. J. Hogan, C. Huang, R. Ischebeck, R. Iverson, C. Joshi, T. Katsouleas, N. Kirby, W. Lu, K. A. Marsh, W. B. Mori, P. Muggli, E. Oz, R. H. Siemann, D. Walz, and M. Zhou, Nature 445, 741 (2007).

${ }^{4}$ M. Litos, E. Adli, W. An, C. I. Clarke, C. E. Clayton, S. Corde, J. P. Delahaye, R. J. England, A. S. Fisher, J. Frederico, S. Gessner, S. Z. Green, M. J. Hogan, C. Joshi, W. Lu, K. A. Marsh, W. B. Mori, P. Muggli, N. Vafaei-Najafabadi, D. Walz, G. White, Z. Wu, V. Yakimenko, and G. Yocky, Nature 515, 92 (2014).
${ }^{5}$ D. H. Whittum, W. M. Sharp, S. S. Yu, M. Lampe, and G. Joyce, Phys. Rev. Lett. 67, 991 (1991).

${ }^{6}$ C. B. Schroeder, C. Benedetti, E. Esarey, F. J. Grüner, and W. P. Leemans, Phys. Rev. E 86, 026402 (2012).

${ }^{7}$ J. B. Rosenzweig, B. Breizman, T. Katsouleas, and J. J. Su, Phys. Rev. A 44, R6189 (1991).

${ }^{8}$ C. Huang, W. Lu, M. Zhou, C. E. Clayton, C. Joshi, W. B. Mori, P. Muggli, S. Deng, E. Oz, T. Katsouleas, M. J. Hogan, I. Blumenfeld, F. J. Decker, R. Ischebeck, R. H. Iverson, N. A. Kirby, and D. Walz, Phys. Rev. Lett. 99, 255001 (2007).

${ }^{9}$ T. J. Mehrling, R. A. Fonseca, A. Martinez de la Ossa, and J. Vieira, Phys. Rev. Lett. 118, 174801 (2017).

${ }^{10}$ J. Vieira, W. B. Mori, and P. Muggli, Phys. Rev. Lett. 112, 205001 (2014).

${ }^{11}$ V. E. Balakin, A. V. Novokhatsky, and V. P. Smirnov, in Proceedings of the 12th International Conference on High-Energy Accelerators, HEACC 1983, Fermilab, Batavia, 11-16 August 1983, C830811 (1983), p. 119.

${ }^{12}$ T. Mehrling, C. Benedetti, C. B. Schroeder, and J. Osterhoff, Plasma Phys. Controlled Fusion 56, 084012 (2014).

${ }^{13}$ P. Sprangle, E. Esarey, and A. Ting, Phys. Rev. A 41, 4463 (1990).

${ }^{14}$ P. Sprangle, E. Esarey, and A. Ting, Phys. Rev. Lett. 64, 2011 (1990).

${ }^{15}$ A. Ting, E. Esarey, and P. Sprangle, Phys. Fluids B: Plasma Phys. 2, 1390 (1990).

${ }^{16}$ P. Mora, J. Thomas, and M. Antonsen, Phys. Plasmas 4, 217 (1997).

${ }^{17}$ The Vlasov equation in the quasi-static approximation was also used in $\mathrm{T}$. Wang, V. Khudik, B. Breizman, and G. Shvets, Phys. Plasmas 24, 103117 (2017) to compute the wakefields in a blowout driven by an infinitesimally short electron beam.

${ }^{18}$ S. A. Yi, V. Khudik, C. Siemon, and G. Shvets, Phys. Plasmas 20, 013108 (2013).

${ }^{19}$ W. Lu, C. Huang, M. Zhou, W. B. Mori, and T. Katsouleas, Phys. Rev. Lett. 96, 165002 (2006).

${ }^{20}$ A. A. Golovanov, I. Y. Kostyukov, J. Thomas, and A. Pukhov, Phys. Plasmas 24, 103104 (2017).

${ }^{21}$ A. Martinez de la Ossa, Z. Hu, M. J. V. Streeter, T. J. Mehrling, O. Kononenko, B. Sheeran, and J. Osterhoff, Phys. Rev. Accel. Beams 20, 091301 (2017).

${ }^{22}$ X. L. Xu, F. Li, W. An, T. N. Dalichaouch, P. Yu, W. Lu, C. Joshi, and W. B. Mori, Phys. Rev. Accel. Beams 20, 111303 (2017). 